\title{
Interleukin-22 drives nitric oxide-dependent DNA damage and dysplasia in a murine model of colitis-associated cancer
}

C Wang ${ }^{1,5}$, G Gong ${ }^{2,5}$, A Sheh ${ }^{1}$, S Muthupalani ${ }^{1}$, EM Bryant ${ }^{1}$, DA Puglisi ${ }^{1}$, H Holcombe ${ }^{1}$, EA Conaway ${ }^{3}$, NA P Parry ${ }^{1}$, V Bakthavatchalu ${ }^{1}$, SP Short ${ }^{4}$, CS Williams ${ }^{4}$, GN Wogan ${ }^{2}$, SR Tannenbaum ${ }^{2,6}$, JG Fox $^{1,2,6}$ and $\mathrm{BH}$ Horwitz ${ }^{3,6}$

The risk of colon cancer is increased in patients with Crohn's disease and ulcerative colitis. Inflammation-induced DNA damage could be an important link between inflammation and cancer, although the pathways that link inflammation and DNA damage are incompletely defined. RAG2-deficient mice infected with Helicobacter hepaticus $(\mathrm{Hh})$ develop colitis that progresses to lower bowel cancer. This process depends on nitric oxide (NO), a molecule with known mutagenic potential. We have previously hypothesized that production of $\mathrm{NO}$ by macrophages could be essential for $\mathrm{Hh}$-driven carcinogenesis, however, whether Hh infection induces DNA damage in this model and whether this depends on NO has not been determined. Here we demonstrate that Hh infection of RAG2-deficient mice rapidly induces expression of iNOS and the development of DNA double-stranded breaks (DSBs) specifically in proliferating crypt epithelial cells. Generation of DSBs depended on iNOS activity, and further, induction of iNOS, the generation of DSBs, and the subsequent development of dysplasia were inhibited by depletion of the Hh-induced cytokine IL-22. These results demonstrate a strong association between Hh-induced DNA damage and the development of dysplasia, and further suggest that IL-22-dependent induction of iNOS within crypt epithelial cells rather than macrophages is a driving force in this process.

\section{INTRODUCTION}

Inflammatory bowel disease (IBD), including Crohn's disease (CD) and ulcerative colitis, affect 1-1.3 million people in the United States, ${ }^{1}$ and patients with IBD are at increased risk of developing colon cancer. ${ }^{2,3}$ It has been hypothesized that inflammation-induced DNA damage leads to mutations that drive carcinogenesis, ${ }^{4}$ and within the gastrointestinal tract, microflora are thought to play a key role in initiating the inflammatory pathways that contribute to the development of cancer. ${ }^{5}$ While definitive associations with specific microflora and lower bowel cancer in patients with IBD have not been identified, DNA damage and genetic mutation are prominent disease components. ${ }^{6}$ Colonization of colitis-prone murine models with enterohepatic Helicobacter species, including Helicobacter hepaticus (Hh), has been strongly associated with the development of colitis and cancer, ${ }^{7}$ and these models are powerful systems with which to study the mechanistic basis for inflammation-induced carcinogenesis within the lower bowel.

Evidence of reactive oxygen and nitrogen-mediated stress is evident in the lower bowel of both patients with IBD and Hh-infected RAG mice. Hh infection increases the production of nitric oxide (NO) in 129RAG2 $2^{-1-}$ mice and immunohistochemistry localized iNOS production to both

${ }^{1}$ Division of Comparative Medicine, Massachusetts Institute of Technology, Cambridge, Massachusetts, USA. ${ }^{2}$ Department of Biological Engineering, Massachusetts Institute of Technology, Cambridge, Massachusetts, USA. ${ }^{3}$ Department of Pathology, Brigham and Women's Hospital, Boston, Massachusetts, USA and ${ }^{4}$ Department of Medicine, Division of Gastroenterology, Hepatology, and Nutrition, and Department of Cancer Biology, Vanderbilt University Medical School, Nashville, Tennessee, USA. Correspondence: BH Horwitz (bhorwitz@partners.org)

${ }^{5}$ These authors contributed equally to this work.

${ }^{6}$ These authors share senior authorship.

Received 14 August 2016; revised 21 December 2016; accepted 3 January 2017; published online 15 February 2017. doi:10.1038/mi.2017.9 
intestinal epithelial cells and infiltrating macrophages 3-4 months after infection. ${ }^{8}$ Treatment of Hh-infected mice with the iNOS inhibitor N-methyl-arginine (NMA) resulted in a trend toward decreased dysplasia and cancer, suggesting that Hh-induced NO production has an important role in this model. ${ }^{8}$ While NO can cause DNA damage, whether NO induces DNA damage in epithelial cells following Hh infection is not known. Given the important parallels between lower bowel pathology that develops in Hh-infected 129RAG2 ${ }^{-1-}$ mice and human patients with IBD, determining whether Hh infection causes DNA damage in intestinal epithelial cells has important implications for the etiology of colitis-associated cancer.

Chronic infection studies have demonstrated that while $\mathrm{Hh}$ is able to persistently colonize the lower bowel of wild type strains, development of colitis and colon carcinoma is strongly enhanced in strains lacking key immunoregulatory factors including IL-10 and regulatory T cells. ${ }^{9}$ Targeted infection of 129RAG2 ${ }^{-1-}$ mice with $\mathrm{Hh}$ has demonstrated that colitis and cancer develop in the absence of B and T lymphocytes. ${ }^{9}$ In contrast, recently described IL-17 and IL-22 producing type 3 innate lymphoid cells appear to play a critical role in Hhinduced innate colitis, as depletion of innate lymphoid cells with anti-Thy1 antibody inhibits disease in this model. ${ }^{10}$ Thus, innate lymphoid cells, and/or their cytokine products, are intimately involved in the intestinal response to Hh infection.

We have found that IL-22 is highly induced within the colon of 129RAG2 $2^{-1-}$ mice following Hh infection. ${ }^{11}$ The IL-22 receptor is expressed on non-hematopoietic cell types, such as epithelial cells, and induces activation of STAT3, ${ }^{12}$ and the ability of IL-22 to induce production of anti-microbial peptides, such as Reg3 $\gamma$, by intestinal epithelial cells play a role in protection from Citrobacter rodentium-induced lower bowel inflammation. ${ }^{13}$ The ability of IL-22 to regulate intestinal microbiota is supported by observations that the gut microbiota are significantly altered in mice lacking IL-22. ${ }^{14}$

While IL-22 has a protective role in the acute response to intestinal pathogens, its role in modulating chronic intestinal inflammatory responses and cancer remains enigmatic. Previous experiments using a chronic model of inflammation-associated cancer induced by $\mathrm{Hh}$ infection followed by treatment with 2-azoxymethane (AOM) demonstrated that treatment with anti-Thy1 antibody or anti-IL-22 antibody for 8 weeks significantly reduced high-grade hyperplasia and invasive colorectal cancer. ${ }^{10}$ Treatment with anti-IL-22 antibody in this model was also associated with reduced STAT3 activation in intestinal epithelium as well as reduced expression of anti-microbial peptide mRNA within epithelial cells. ${ }^{10}$ However, to our knowledge, whether IL-22 is directly involved in modulating key mutagenic pathways, such as the induction of DNA damage, that are thought to play a causative role in the development of inflammation-associated cancers has not been previously assessed. Therefore, in this study, we examined the ability of $\mathrm{Hh}$ to induce DNA damage following $\mathrm{Hh}$ infection, and further, evaluated the role of IL-22 and $\mathrm{NO}$ in this process.

\section{RESULTS}

\section{Hh infection rapidly induces inflammatory pathology within} the large bowel

Frank malignancy in Hh-susceptible models typically does not develop until several months post infection. ${ }^{7}$ Previous studies have evaluated Hh-induced inflammatory changes within the lower bowel of RAG2-deficient mice at 6 weeks or longer following infection, or at 12 and 20 weeks post infection (WPI) in mice subsequently treated with $\mathrm{AOM} .^{10,11,15}$ However, Hh-induced inflammatory changes at early time points following infection, such as 2 weeks, have not been characterized. We reasoned that evaluating $\mathrm{Hh}$-induced inflammatory responses and lower bowel epithelial damage at earlier time points might distinguish key pathways involved in inflammatory-associated carcinogenesis. We therefore infected male and female adult 129RAG2 $2^{-1-}$ mice with Hh by oral gavage and evaluated gross and histologic alterations at 2 and 6 WPI. Tissue sections stained with hematoxylin and eosin from the cecum, proximal, transverse, and distal colon were scored by board-certified veterinary pathologists for histomorphological features, including inflammation, edema, epithelial defects, crypt atrophy, hyperplasia, and dysplasia/neoplasia (Figure 1a). The cumulative scores were represented as a histological activity index (HAI) (Figure 1b). At 2 WPI, the infected bowel exhibited significant inflammatory pathology that was most intense in the cecum with decreasing intensity in the ascending, transverse, and descending colon. There was mild to moderate mucosal and submucosal inflammation characterized chiefly by neutrophils and macrophages. Further, there was increased crypt epithelial mitotic activity in association with mild to moderate epithelial hyperplasia. In addition, there was low to moderate epithelial dysplastic changes which were most notable in the vicinity of inflammatory foci, although these dysplastic changes did not reach the levels of high-grade dysplasia/glandular intraepithelial neoplasia or carcinoma. The overall histological findings within bowel segments from mice evaluated at 6 WPI were largely similar to findings at 2 WPI (Figure 1a and $\mathbf{b}$ ). Further, we found significant induction of mRNA for both IL-22 and tumor necrosis factor within total RNA isolated from the cecum and colon (Figure 1c). Interestingly, Hh infection was associated with increases in the relative percentages of inflammatory macrophages $\left(\mathrm{MHCII}^{+} \mathrm{Ly}_{6 \mathrm{C}}{ }^{+}\right)$and decreases in the percentages of anti-inflammatory $\mathrm{MHCII}^{+} \mathrm{Ly}_{6} \mathrm{C}^{-}$macrophages within the $\mathrm{CD}_{11 \mathrm{~b}^{+}} \mathrm{CD} 4^{+}$cell gate (Supplementary Figure S1). These results indicate that $\mathrm{Hh}$ infection induces inflammation and induction of IL-22 as early as 2 weeks after infection associated with expansion of inflammatory macrophage populations. Therefore, the 2-week time point was used for detailed sampling in subsequent experiments.

\section{Hh-infection induces iNOS activity within colonic epithelial cells}

Mice chronically infected with $\mathrm{Hh}$ demonstrate marked induction of iNOS within the colon as well as increases in urinary excretion of nitrate, suggesting increased production of NO. ${ }^{8}$ To examine whether iNOS was expressed within the lower 

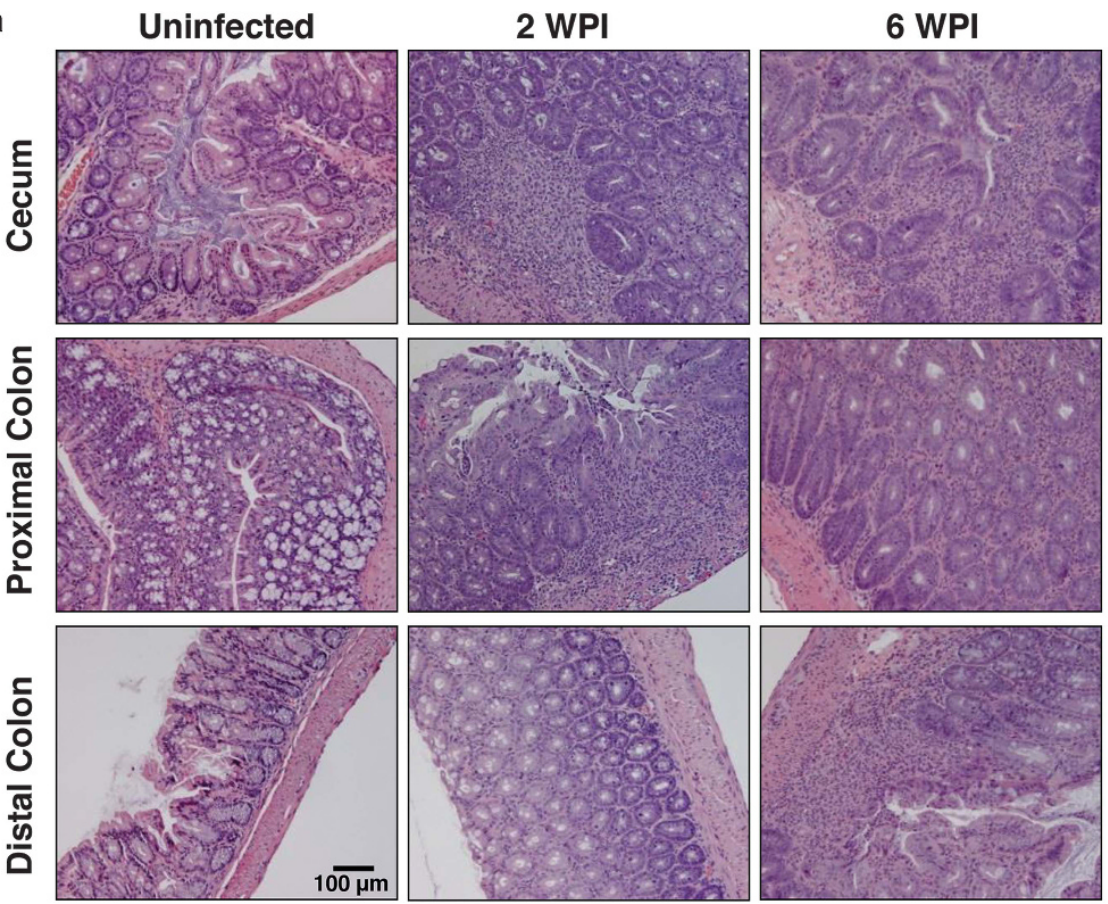

b

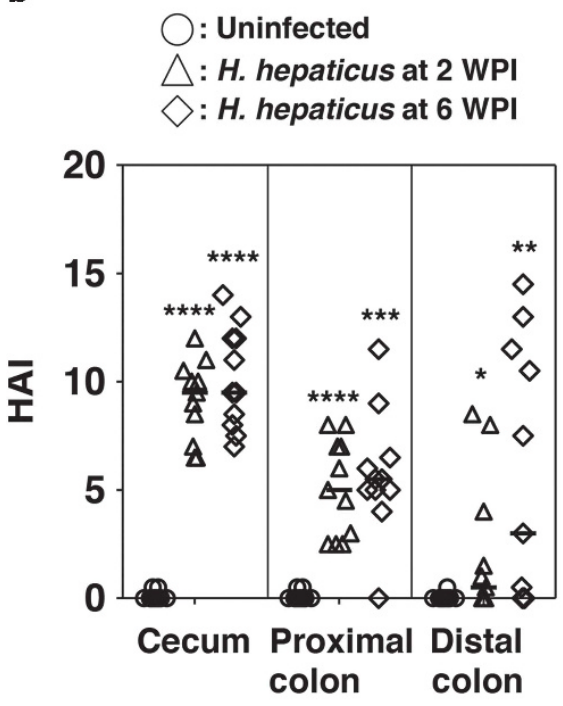

c
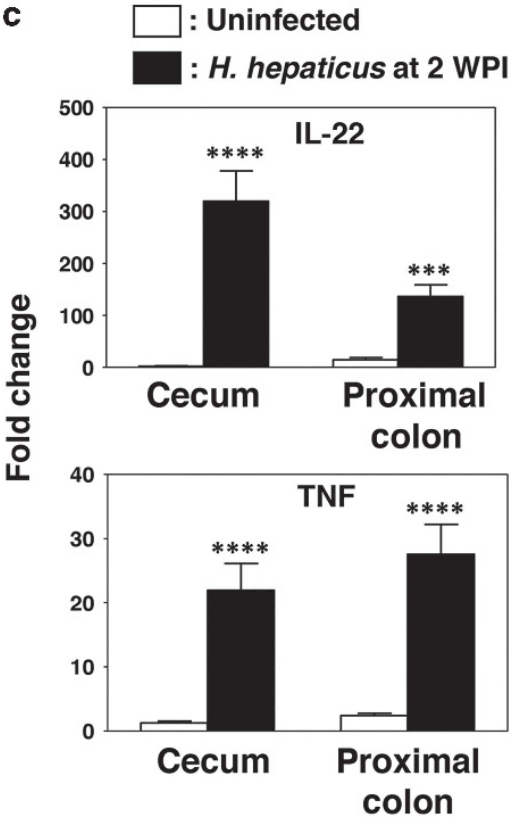

Figure $1 \mathrm{Hh}$ infection induces acute inflammation in the lower bowel. Uninfected $129 \mathrm{RAG}^{-/-}$mice or $129 \mathrm{RAG} 2^{-/-}$mice infected with Hh by oral gavage were killed at 2 WPI and 6 WPI. Sections of the cecum, proximal colon, and distal colon were processed for histological analysis and RNA isolation. (a) Representative hematoxylin and eosin staining of cecum, proximal colon, and distal colon. (b) HAl from individual mice ( $n=10-11)$. (c) RT-PCR results were obtained from RNA isolated from indicated tissue at 2 WPI. $n=17-20 .{ }^{\star} P<0.05,{ }^{\star *} P<0.01,{ }^{* * *} P<0.001$, and ${ }^{* * * *} P<0.0001$.

bowel of 129RAG2 ${ }^{-1-}$ mice at 2 WPI, we compared iNOS expression in total RNA from the cecum of infected and control uninfected mice. We found that there was a $>500$-fold induction of iNOS following $\mathrm{Hh}$ infection (Figure 2a), suggesting that this is an early event mediating $\mathrm{Hh}$ pathogenesis. We have previously found that iNOS protein can be localized within lower bowel epithelial cells as well as infiltrating macrophages in $129 \mathrm{RAG} 2^{-1-}$ mice chronically infected with $\mathrm{Hh}^{8}{ }^{8}$ To extend these results, we performed immunofluorescence staining of cecal sections from mice infected with Hh for 2 weeks as well as uninfected control mice. Remarkably, Hh strongly induced the expression of iNOS along the apical aspect of cecal $\mathrm{EpCAM}^{+}$epithelial cells, while staining within $\mathrm{F} 4 / 80^{+}$macrophages was quite limited (Figure 2b). Further, we observed increases in nitrotyrosine staining (previously associated with elevated levels of $\mathrm{NO}^{16}$ ) within 


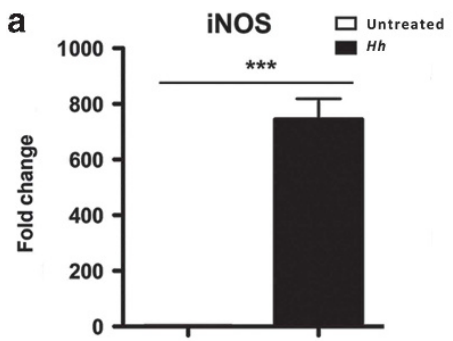

b Uninfected
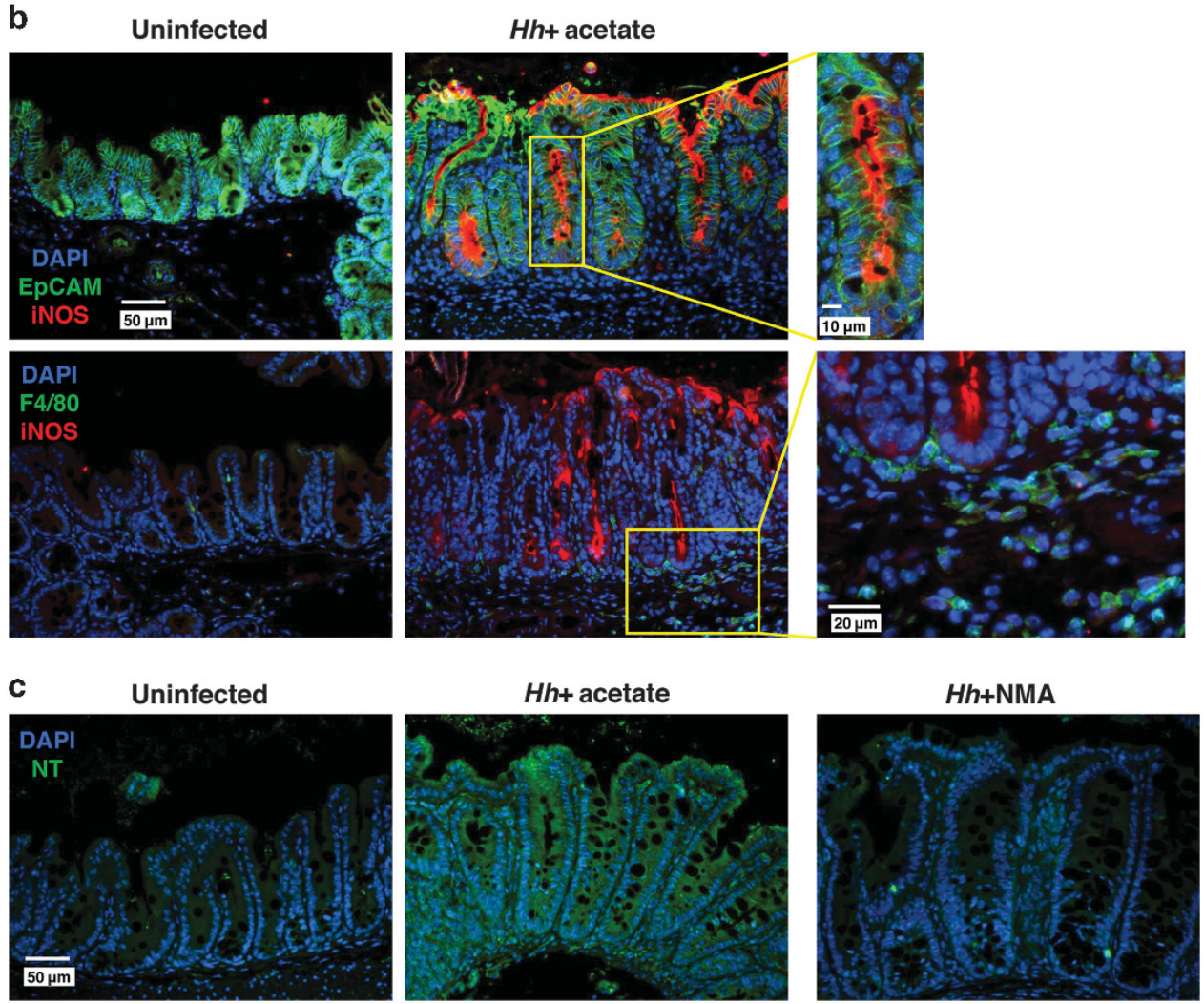

Figure 2 Hh induces expression of iNOS and production of NO within the lower bowel epithelium. (a) RT-PCR results were obtained from RNA isolated from the cecum at 2 WPI, $n=8-11$. (b) Representative sections from uninfected 129RAG2 ${ }^{-1-}$ mice or mice infected with Hh for 2 weeks evaluated for expression of iNOS within the epithelium (EpCAM) or macrophages (F4/80). Boxed areas in center images are shown at higher magnification on right. (c) Representative images from uninfected mice, or infected mice treated either with $\mathrm{N}$-methyl-arginine (NMA) or acetate (control for NMA), evaluated for the presence of nitrotyrosine. ${ }^{* \star *} P<0.001$.

the cecal epithelium of Hh-infected mice compared to uninfected controls, which was inhibited by treatment of infected mice with the iNOS inhibitor NMA for 1 week before killing (Figure 2c). These results indicate that $\mathrm{Hh}$ induces epithelial iNOS expression at early time points after infection, and that this is likely associated with increased levels of NO within epithelial cells. While we were able to appreciate faint iNOS staining of $\mathrm{F} 4 / 80^{+}$macrophages, the considerably stronger epithelial staining raises the possibility that epithelial cells rather than infiltrating myeloid cells are the primary source of NO at this early time point.

\section{Hh-infection increases epithelial crypt cell proliferation and induces DNA damage}

Reactive oxygen and nitrogen species are thought to play a central role in DNA damage and mutagenesis associated with colitis-associated cancer. ${ }^{17}$ We have previously hypothesized that $\mathrm{NO}$ produced following $\mathrm{Hh}$ infection plays a role in the development of malignancy observed in Hh-infected 129RAG2 ${ }^{-1-}$ mice by facilitating DNA damage in intestinal epithelial cells, ${ }^{8}$ although NO-dependent epithelial DNA damage has not previously been directly demonstrated in this model. To assess this possibility, sections of cecum from uninfected control mice and mice infected with $\mathrm{Hh}$ for 2 weeks were stained with Ki67, a marker of cell proliferation, and anti$\gamma \mathrm{H} 2 \mathrm{AX}$, an antibody that recognizes the phosphorylated form of histone $\mathrm{H} 2 \mathrm{AX}$, which is rapidly and transiently assembled on chromatin at the sites of DNA double-stranded breaks (DSBs) ${ }^{18}$ (Figure 3a). As expected, Ki67 staining was largely confined to the base of the cecal crypts in uninfected mice, while staining was more extensive in Hh-infected mice (Figure 3a). This was reflected in increased numbers of $\mathrm{Ki}^{+} 7^{+}$epithelial cells 

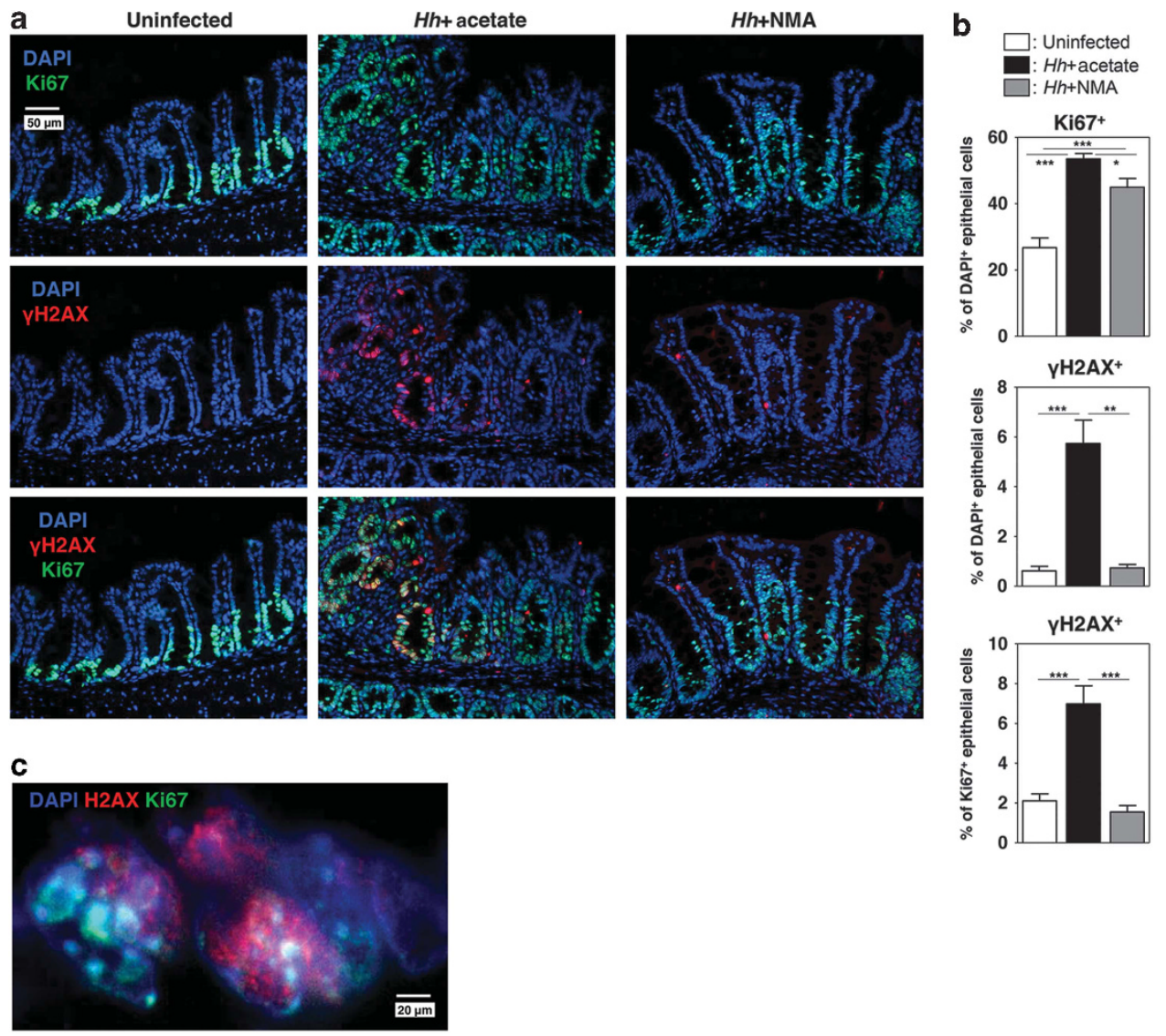

Figure $3 \mathrm{Hh}$ induces iNOS-dependent DNA damage. Uninfected 129RAG2 ${ }^{-1-}$ mice or mice infected with Hh for 2 weeks were treated either with $\mathrm{N}$-methyl-arginine (NMA) or acetate in the drinking water for the final week of infection. (a) Representative histological sections from the cecum were evaluated by immunofluorescence with indicated stains. (b) Bar graphs demonstrating the percent of epithelial cells that were Ki67 ${ }^{+}$(top), the percent of epithelial cells that were $\gamma \mathrm{H}_{2} \mathrm{AX}^{+}$(middle), and the percent of $\mathrm{Ki}^{+} 7^{+}$epithelial cells were also $\gamma \mathrm{H} 2 \mathrm{AX}{ }^{+}$(bottom). $n=7-10$. (c) Higher magnification image of cecum from an Hh-infected mouse demonstrating punctate nuclear staining with $\gamma \mathrm{H} 2 \mathrm{AX}$. ${ }^{\star} P<0.05$, ${ }^{\star \star} P<0.01$, and ${ }^{\star \star \star} P<0.001$.

following Hh infection (Figure 3b). Interestingly, while there was limited staining for $\gamma \mathrm{H} 2 \mathrm{AX}$ within epithelial cells from uninfected mice, $\gamma \mathrm{H} 2 \mathrm{AX}^{+}$epithelial cells were frequently found within the crypts of infected mice (Figure 3a). $\gamma \mathrm{H} 2 \mathrm{AX}$ staining was largely restricted to $\mathrm{Ki}^{+} 7^{+}$cells, which was reflected in a statistically significant increase in the percent of $\mathrm{Ki}^{+}{ }^{+}$-positive cells that were also $\gamma \mathrm{H} 2 \mathrm{AX}^{+}$following $\mathrm{Hh}$ infection (Figure $3 \mathbf{b}$ ). $\gamma \mathrm{H} 2 \mathrm{AX}$ staining exhibited punctate nuclear staining within epithelial cells of $\mathrm{Hh}$-infected mice (Figure 3c), consistent with the previously described staining pattern for labeling DNA DSBs. Remarkably, NMA treatment significantly decreased the percentage of $\mathrm{Ki}^{+}{ }^{+}$cells that were $\gamma \mathrm{H}_{2} \mathrm{AX}^{+}$in Hh-infected mice (Figure 3a and $\mathbf{b}$ ). These results strongly suggest that NO-dependent DNA damage is the result of Hh-induced epithelial iNOS expression.

\section{Hh-induced iNOS expression is mediated by IL-22}

iNOS induction within myeloid cells is associated with inflammatory factors, such as lipopolysaccharide and tumor necrosis factor, that induce NF- $\kappa \mathrm{B}$ activation. ${ }^{19}$ However, pathways responsible for iNOS induction in epithelial cells are less well defined. It has been reported that IL-22 can synergize with IFN- $\gamma$ to induce iNOS in cultured epithelial cells. ${ }^{20}$ This process depended on STAT3 activation, but whether this pathway plays an important role in inducing epithelial iNOS expression in vivo following $\mathrm{Hh}$ infection has not been determined. To examine this question, we treated Hh-infected 129RAG2 ${ }^{-1-}$ mice with a previously validated depleting antiIL-22 antibody ${ }^{10}$ or a control antibody during the final week of the 2-week infection protocol. As expected, Hh infection induced expression of iNOS, as well as the IL-22 target genes anti-microbial peptides $\operatorname{Reg} 3 \beta$ and $\operatorname{Reg} 3 \gamma$ (Figure 4a). Depletion of IL-22 strongly inhibited induction of Reg $3 \beta$ and Reg3 $\gamma$ (Figure 4a), and inhibited STAT3 activation within total colonic extracts (Figure $4 \mathrm{~b}$ ) indicating successful depletion of IL-22 activity, and suggesting that the marked increase in IL-22 mRNA observed in this model results in the production of functional protein. Remarkably, depletion of IL-22 also inhibited the induction of iNOS mRNA (Figure 4a), and strongly reduced iNOS staining on the epithelial apical border following Hh infection (Figure 4c), as well as reducing the percentage of crypts that demonstrated positive staining with iNOS (Figure 4d). The loss of iNOS staining was accompanied by decreased staining with anti-nitrotyrosine antibody, suggesting decreased production of NO (Figure 4c). Interestingly, Hh infection also induced nuclear staining with antibody 
a

음
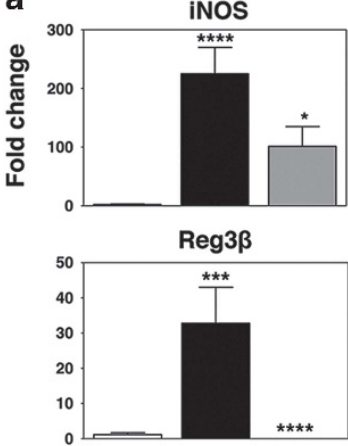

C
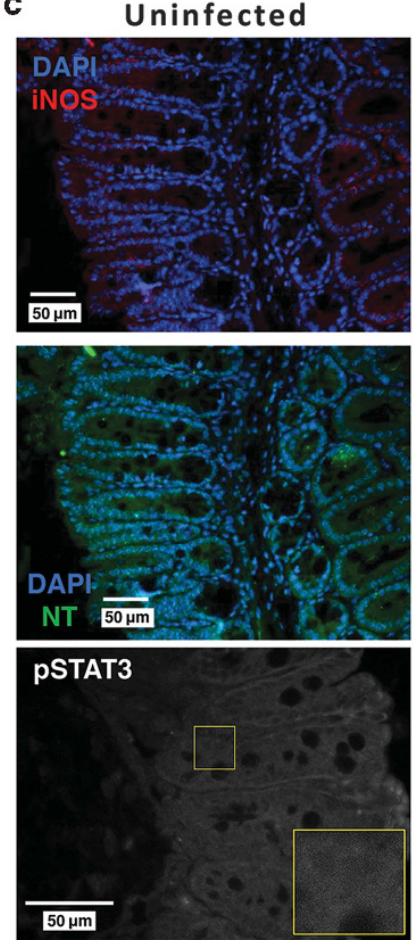

d

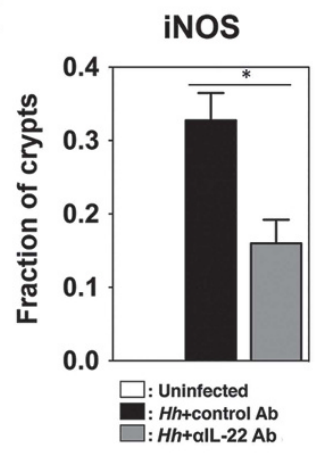

b

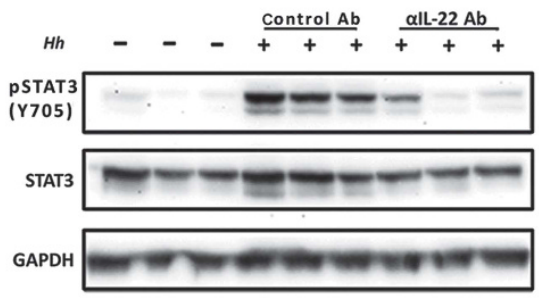

: Hh+control Ab

: Hh+alL-22 Ab
Reg3y

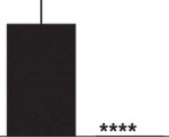

Uninfected
$H h+$ control Ab
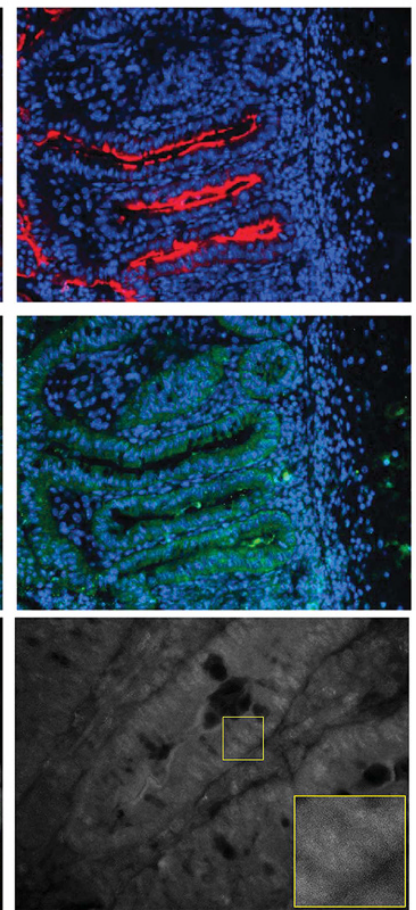

$H h+\alpha I L-22 A b$
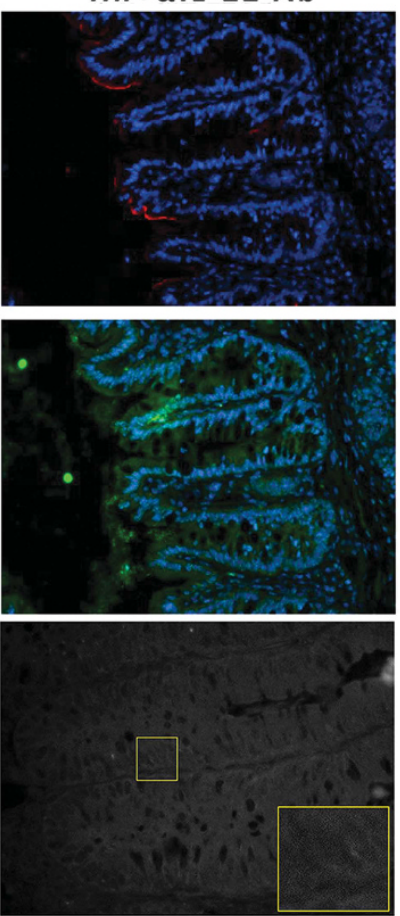

e

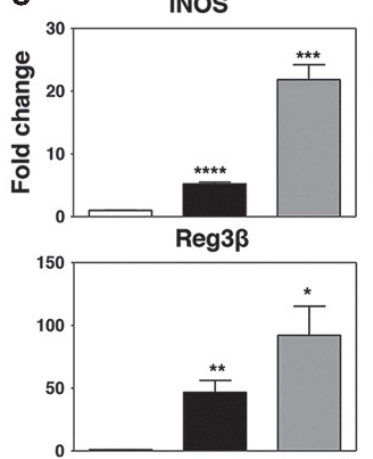

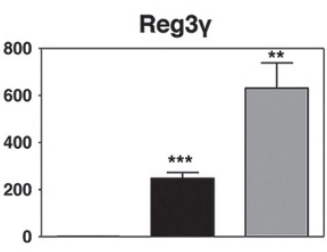

$\square$ : Untreated

1 ng IL-22

: 5 ng IL-22

Figure 4 IL-22 is necessary for induction of iNOS. Uninfected $129 \mathrm{RAG} 2^{-1-}$ mice or mice infected with $\mathrm{Hh}$ for 2 weeks were treated either with $100 \mu \mathrm{g}$ control Ab or anti-IL-22 Ab every other day during the second week of infection. (a) RNA was isolated from the cecum and expression of indicated gene was analyzed by RT-PCR. $n=9-11$. (b) Western blot of total colonic extracts from three individual mice per group with indicated antibodies. (c) Representative histological sections from the cecum were analyzed with indicated stains. (d) The fraction of crypts that exhibited iNOS staining was compared $(n=3-4)$. (e) RNA isolated from mouse intestinal organoids treated with IL-22 at indicated dosages was analyzed by RT-PCR. $n=6$. ${ }^{*} P<0.05$, ${ }^{\star *} P<0.01,{ }^{* \star *} P<0.001$, and ${ }^{\star \star * \star} P<0.0001$. 

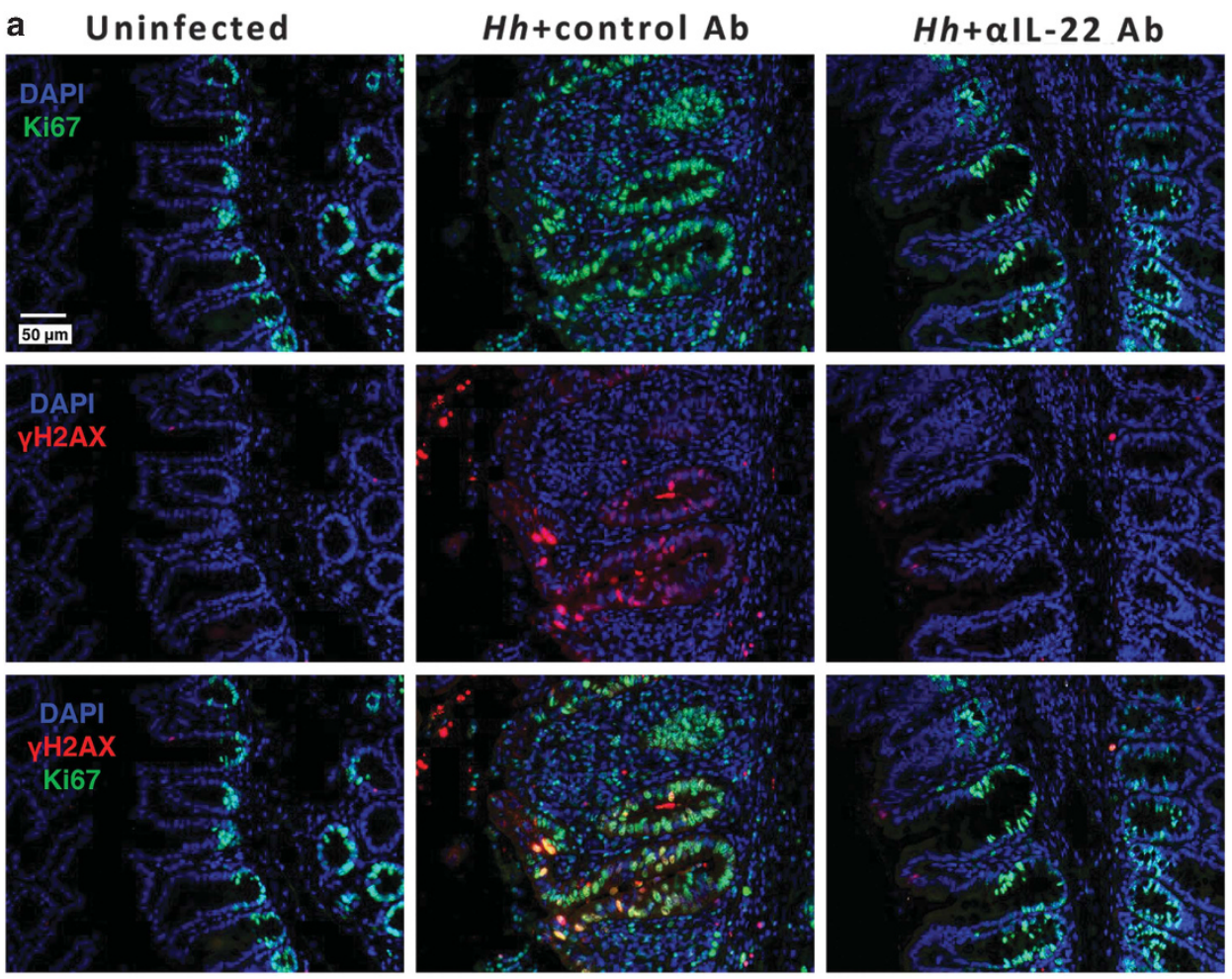

b
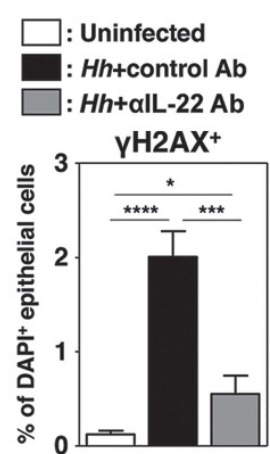
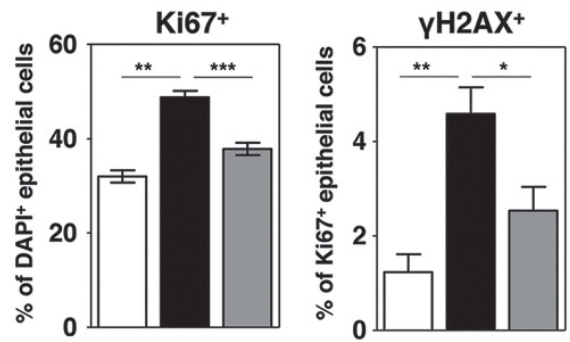

C $\bigcirc:$ Uninfected

$\triangle:$ Hh+control Ab

$\diamond: H h+\alpha I L-22 A b$

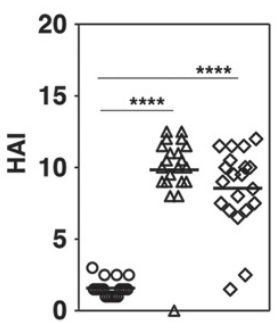

Figure 5 Hh-induced DNA damage depends on IL-22. Uninfected 129RAG2 ${ }^{-1}$ - mice or mice infected with Hh for 2 weeks were treated either with depleting IL-22 Ab or a control Ab. (a) Representative histological sections from the cecum were analyzed with indicated stains. (b) Graphs representing the percent of epithelial cells that were $\gamma \mathrm{H} 2 \mathrm{AX}^{+}$(left), the percent of epithelial cells that were $\mathrm{Ki} 7^{+}$(center), and the percent of Ki67 ${ }^{+}$epithelial cells that were also $\gamma \mathrm{H} 2 \mathrm{AX}^{+}$(right) in indicated groups. $n=8-14$. (c) HAl of individual mice from each group. ${ }^{\star} P<0.05$, ${ }^{\star \star} P<0.01,{ }^{\star * \star} P<0.001$, and ${ }^{* \star \star \star} P<0.0001$.

directed at pY705-STAT3 within crypt epithelial cells that was blocked by anti-IL-22 antibody (Figure 4c), correlating with results obtained by western blotting. To determine whether IL-22 was capable of inducing iNOS expression directly in intestinal epithelial cells, wild type mouse enteroids were treated with $1 \mathrm{ng}$ or $5 \mathrm{ng}$ of IL-22. We observed a significant and dose-dependent induction of Reg3 $\beta$ and $\operatorname{Reg} 3 \gamma$, as well as iNOS (Figure 4e). These results strongly suggest that Hh-induced IL-22 directly induces STAT3 activation, expression of iNOS, and production of NO within epithelial cells of the lower bowel.

\section{Hh-induced DNA damage depends on IL-22}

The observations that induction of iNOS in this model depends on IL-22 raised the possibility that IL-22 was necessary for
Hh-induced epithelial DNA damage. To directly test this hypothesis, we evaluated $\gamma \mathrm{H} 2 \mathrm{AX}$ staining in Hh-infected mice following depletion of IL-22. Notably, IL-22 depletion markedly inhibited staining with $\gamma \mathrm{H} 2 \mathrm{AX}$ (Figure 5a and $\mathbf{b}$ ). While IL-22 depletion appeared to partially inhibit Hh-induced increases in epithelial cells that stain positively for Ki67 (Figure 5a and b), the percentage of $\mathrm{Ki}^{+} 7^{+}$epithelial cells that also stain positively for $\gamma \mathrm{H} 2 \mathrm{AX}$, was significantly lower in Hh-infected mice treated with anti-IL-22 antibody than with the control antibody (Figure 5b), indicating the decrease in epithelial cells staining positively for $\gamma \mathrm{H} 2 \mathrm{AX}$ is not simply the result of fewer proliferating epithelial cells following IL-22 depletion. Further, we did not appreciate significant differences in overall HAI scores in the cecum following treatment with the anti-IL-22 antibody (Figure 5c), suggesting that effects of IL-22 
a

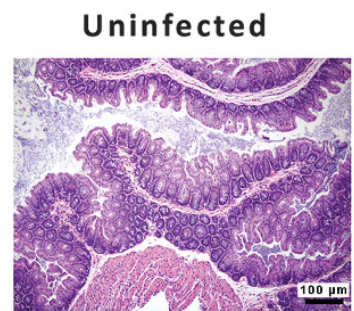

\section{Cecum}

$H h+$ control $A b$

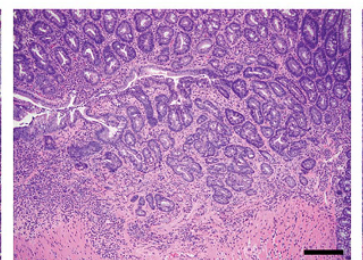

$H h+\alpha I L-22 A b$

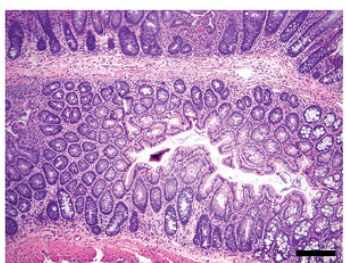

b

$\bigcirc:$ Uninfected
$\triangle:$ Hh+control Ab

$\diamond: H h+a I L-22 \mathrm{Ab}$

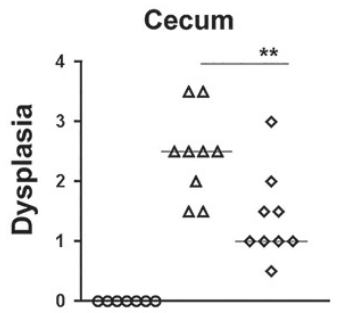

Colon
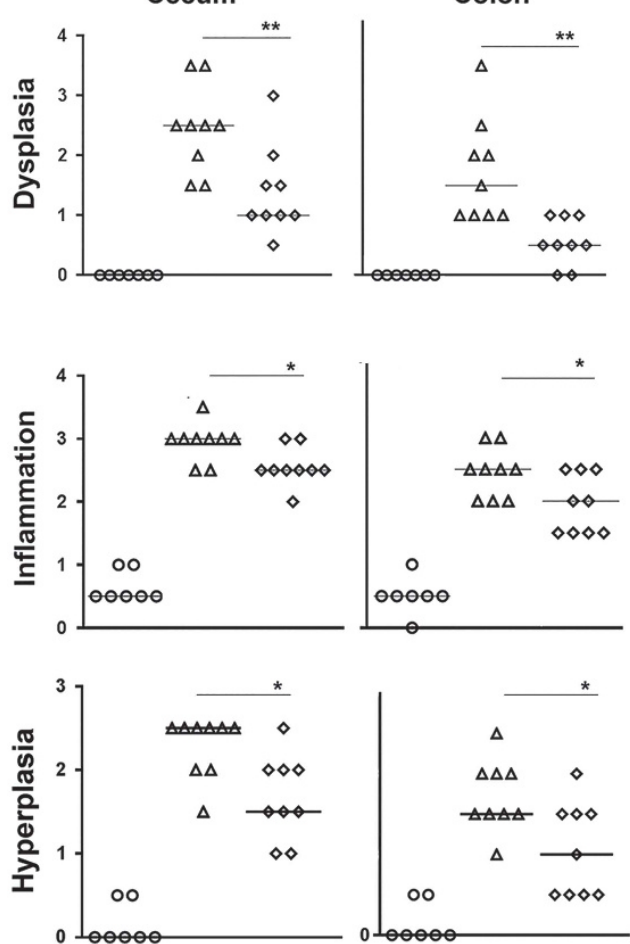

C

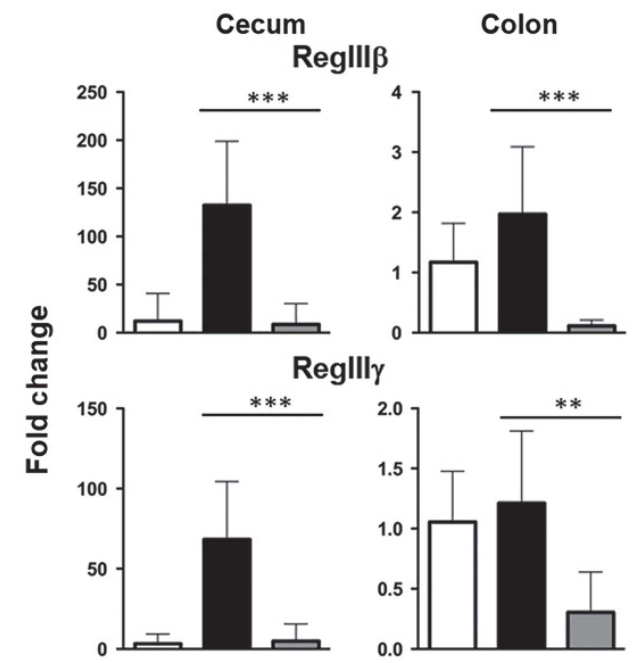

iNOS
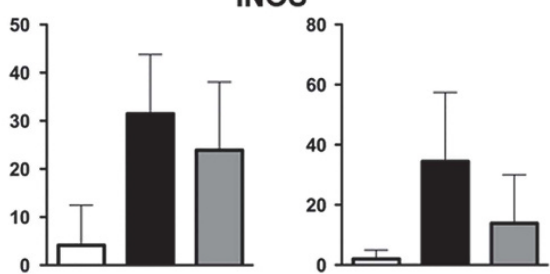

Figure 6 IL-22 depletion inhibits dysplasia in mice chronically infected with Hh. (a) Representative hematoxylin and eosin staining of cecum from uninfected 129RAG2 ${ }^{-1-}$ mice or mice infected with $\mathrm{Hh}$ for 10 weeks treated either with control or IL-22 depleting Ab for the final week of infection, as indicated. (b) Inflammation, hyperplasia, and dysplasia scores for uninfected mice $(n=7)$ or mice infected with $\mathrm{Hh}$ for 10 weeks and treated with control $\mathrm{Ab}(n=8)$ or anti-IL-22 depleting antibody $(n=8)$ as above. (c) Expression of RegllI $\beta$, Reglll $\gamma$, and iNOS in total RNA isolated from cecum or colon of mice treated as above $(n=7-8) .{ }^{\star} P<0.05,{ }^{\star *} P<0.01$, and ${ }^{* * *} P<0.001$.

depletion on DNA damage are not due to differences in overall levels of inflammation.

\section{IL-22 depletion inhibits DNA damage and dysplasia in 129RAG2 ${ }^{-l-}$ mice chronically infected with Hh}

We have demonstrated that $\mathrm{Hh}$ infection induces IL-22dependent DNA damage in the rapid infection model. However, as dysplasia and cancer are not features of this early time point, we were unable to determine whether IL-22 was necessary for the development of cancer. To evaluate this issue, 129RAG2 $2^{-1-}$ mice were infected with $\mathrm{Hh}$ for 10 weeks and treated with either depleting IL-22 antibody or control antibody for the final week of infection. Hh-infected mice treated with control Ab, developed colitis that was characterized by inflammation, hyperplasia, and significant dysplasia.
IL-22 depletion significantly inhibited these parameters and most notably had a marked inhibitory effect on the development of dysplasia (Figure 6a and $\mathbf{b}$ ). Consistent with effective depletion of IL-22, evaluation of gene expression from total RNA isolated from the cecum and colon demonstrated significant reduction of anti-microbial gene expression in the group treated with the IL-22-depleting Ab compared to the group treated with the control $\mathrm{Ab}$, although we did not detect statistically significant differences in expression of iNOS (Figure 6c).

In conjunction with dysplasia, we found that crypt epithelial cells in mice infected with Hh for 10 weeks and treated with control antibody demonstrated widespread punctate nuclear staining for $\gamma \mathrm{H} 2 \mathrm{AX}$, indicating significant DNA damage (Figure 7a). As in the $\gamma \mathrm{H} 2 \mathrm{AX}$ staining that was observed 2 
a

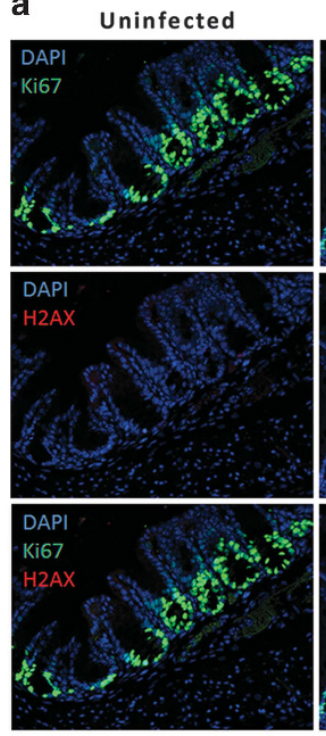

C
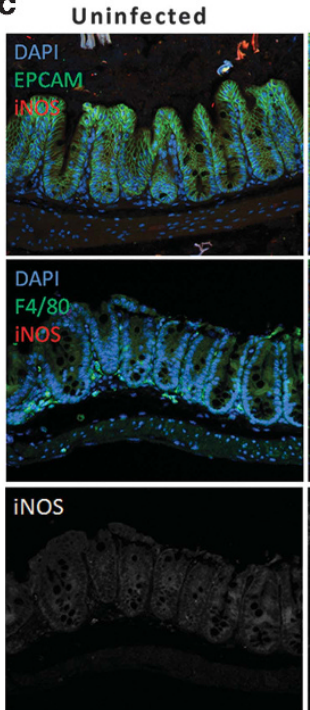

$H h+$ control $\mathrm{Ab}$
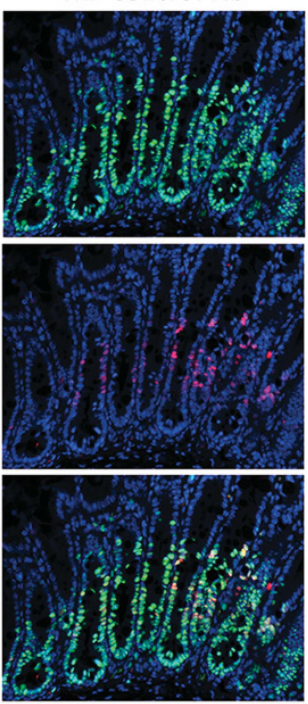

$H h+$ control $\mathrm{Ab}$
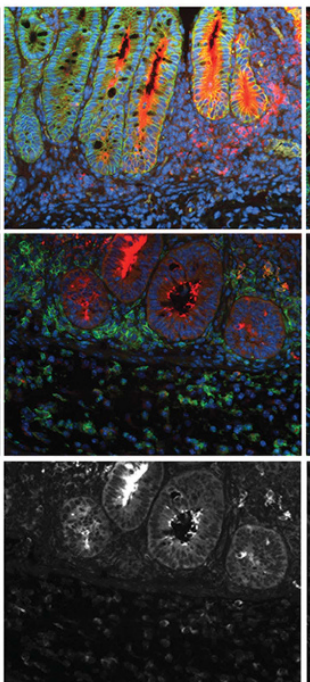

$H h+\alpha I L-22 A b$
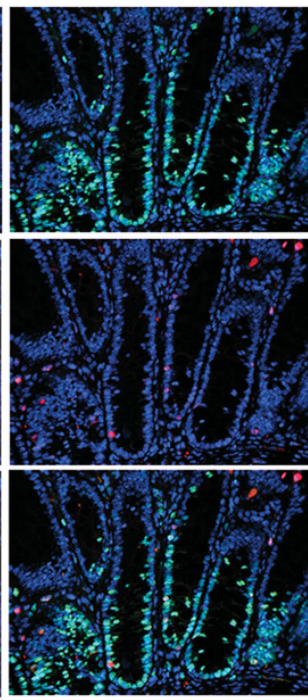

$H h+\alpha I L-22 A b$

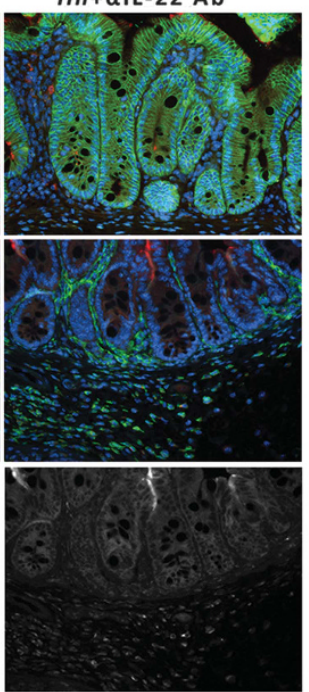

b $\square$ : Uninfected

: Hh+control Ab
: Hh+alL-22 Ab

Ki67

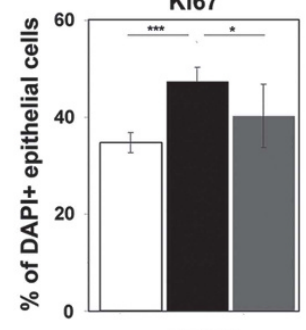

H2AX

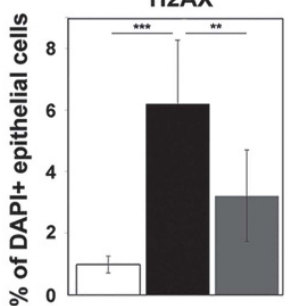

d

$\square$ : Uninfected

: Hh+control Ab

: Hh+alL-22 Ab

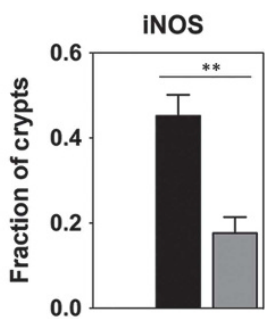

Figure 7 IL-22 depletion inhibits DNA damage in mice chronically infected with Hh. Uninfected 129RAG2 ${ }^{-1-}$ mice or mice infected with Hh for 10 weeks were treated either with depleting IL-22 Ab or a control Ab for the final week of the experiment. (a) Representative histological sections from the cecum were analyzed with indicated stains. (b) Graphs representing the percent of epithelial cells that were Ki67 ${ }^{+}$(top) or $\gamma \mathrm{H} 2 \mathrm{AX}{ }^{+}$(bottom), $n=9$. (c) Representative histological sections with indicated stains comparing localization of iNOS within epithelial cells (EPCAM) and macrophages (F4/80). Single channel stains of iNOS alone are provided to more clearly demonstrate the differential effects of IL-22 depletion on epithelial cells and macrophages. (d) The fraction of crypts that exhibited iNOS staining was compared $(n=3-4) .{ }^{*} P<0.05,{ }^{* \star} P<0.01$, and ${ }^{* \star *} P<0.001$.

weeks following Hh infection, staining was largely confined to $\mathrm{Ki}^{+} 7^{+}$cells, which were also increased in number, indicating the presence of DSBs in proliferating epithelial cells (Figure 7a and b). Remarkably, depletion of IL-22 reduced Hh-induced staining of iNOS along the apical border of crypt epithelial cells and the percentage of crypts that demonstrated epithelial iNOS staining, without dramatically altering expression within $\mathrm{F} 4 / 80^{+}$macrophages (Figure $7 \mathrm{c}$ and $\mathbf{d}$ ), supporting the hypothesis that IL-22 specifically induces iNOS expression within epithelial cells. These results indicate that depletion of IL-22 inhibits the development of DNA damage and dysplastic changes in this chronic Hh infection model.

\section{Depletion of IL-22 does not interfere with neutrophil recruitment}

Our results suggest that induction of iNOS and subsequent production of NO plays a significant role in mediating IL-22dependent DSBs following Hh infection. However, it has also been suggested that other ROS could play a role in the development of DNA damage following Hh infection. ${ }^{15}$ Neutrophils are a potent source or ROS and accumulate in the colon following Hh infection, and it has been recently shown that treatment of $129 / \mathrm{RAG}^{-/-}$mice with anti-Thy1 antibody interferes with neutrophil recruitment to the colon 2 weeks after infection. ${ }^{21}$ As Thyl antibody depletes innate 
a
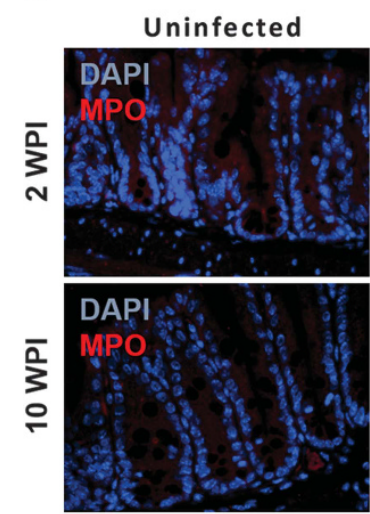

$H h+$ control $\mathrm{Ab}$
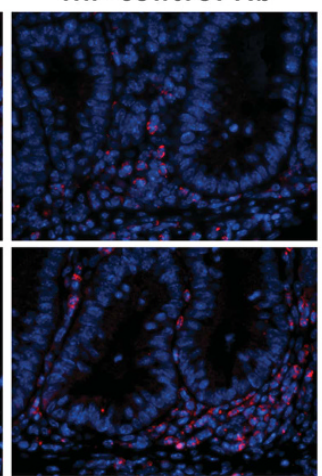
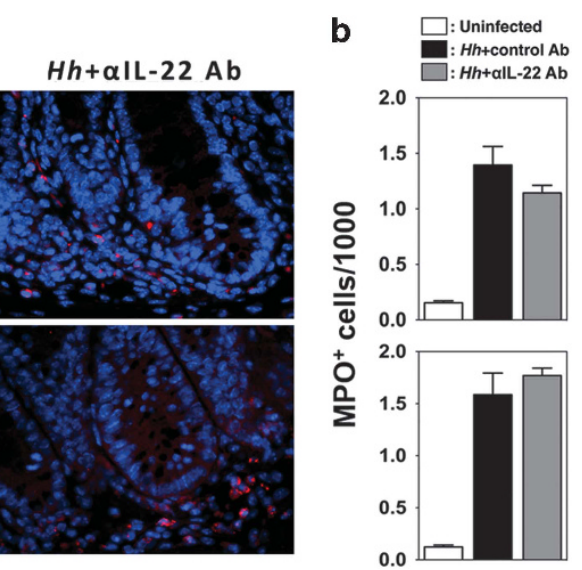

Figure 8 IL-22 depletion does not inhibit neutrophil recruitment to the cecum following Hh infection. (a) Representative histological sections from the cecum of uninfected 129RAG2 ${ }^{-1-}$ mice or mice infected with $\mathrm{Hh}$ for 2 and 10 weeks were treated either with depleting IL-22 Ab or a control Ab for the final week of the experiment. (a) Micrographs show representative sections stained with antibody to MPO. (b) The number of MPO ${ }^{+}$cells per 1,000 cells within the cecum was determined ( $n=4-8$ mice per group).

lymphoid cells from the colon and these cells are a source of IL-22, it raises the possibility that IL-22 depletion could limit the development of DNA damage by interfering with neutrophil recruitment. To evaluate this possibility, we compared the numbers of neutrophils infiltrating the cecum and colon in mice treated with depleting IL-22 antibody or control antibody at both 2 and 10 weeks post Hh infection, using staining for the neutrophil marker myeloperoxidase (MPO). Interestingly, we found no significant difference in the number of infiltrating $\mathrm{MPO}^{+}$neutrophils in mice that received IL-22 antibody at either 2 or 10 WPI (Figure 8a and b). This is consistent with our observations of minimal differences in overall levels of inflammation between IL-22 or control Ab-treated mice. These results indicate that depletion of IL-22 does not interfere with the recruitment of neutrophils in this model, suggesting that a defect in neutrophil recruitment is not responsible for inhibition of DSBs observed following treatment with anti-IL-22.

\section{Hh-induced IL-22 expression exacerbates dysbiosis}

Previous observations indicate that expression of IL-22 can markedly influence colonization levels of intestinal pathogens, ${ }^{13}$ and it has been suggested that the composition of the microbiome can affect the development of intestinal cancer. ${ }^{22}$ To determine whether IL-22 modulates colonization levels of Hh following infection of $129 \mathrm{RAG}^{-1-}$ mice, we used polymerase chain reaction (PCR) to quantify levels of the Hh 16s rRNA gene within cecal content of Hh-infected mice treated with control or anti-IL-22 antibody. ${ }^{23}$ We were unable to observe significant differences at either 2 weeks (Figure 9a) or 10 weeks (data not shown) after infection, suggesting that depletion of IL-22 has minimal influence on the absolute level of Hh colonization. As it has been suggested that colon cancer may be associated with intestinal dysbiosis, ${ }^{24}$ we next used $16 \mathrm{~s}$ rRNA sequencing of bacterial DNA isolated from fecal content to evaluate microbial communities in mice infected with $\mathrm{Hh}$ for 2 weeks. We found that $\mathrm{Hh}$ infection of $129 \mathrm{RAG}^{-/-}$mice induced marked intestinal dysbiosis characterized by significant decreases in Chaol and Shannon diversity indexes (Figure 9b), marked increases in the relative abundances of the phylum Proteobacteria and the family Enterobacteriaceae, as well as decreases in the relative abundances of the phylum Bacteriodetes (Figure 9c). Interestingly, depletion of IL-22 partially ameliorated the Hh-induced dysbiosis, exemplified by increase in diversity indexes, decreases in the relative abundance of Proteobacteria and Enterobacteriaceae, and increases in Bacteriodetes. To confirm these differences, we used phyla and family specific qPCR for Bacteriodetes and Enterobacteriaceae and found results that mirrored results obtained with $16 \mathrm{~S}$ sequencing (Figure 9d). These results indicate that $\mathrm{Hh}$ infection induces a dysbiotic state that is at least in part mediated by IL-22.

\section{DISCUSSION}

In this study, we have demonstrated that Hh infection induces DNA DSBs in proliferating crypt epithelial cells within the lower bowel. The ability of $\mathrm{Hh}$ to induce IL-22 was essential for this process, as was the ability of IL-22 to induce iNOS and production of NO. In addition to blocking the accumulation of DSBs, depletion of IL-22 inhibited the development of dysplasia that develops in chronically infected mice, a lesion that is thought to represent a precursor to frank malignancy. Given the association between DNA damage and malignancy, these results suggest that the ability of IL-22 to drive DSBs during inflammatory responses to intestinal bacteria may be a key factor in the development of colitis-associated cancer.

It has previously been demonstrated that depletion of IL-22 but not IL-17 inhibits dysplasia and invasive adenocarcinoma when evaluated at 20 WPI in Hh-infected 129/RAG2 ${ }^{-1-}$ mice that were treated with the carcinogen AOM. ${ }^{10}$ Further, it has been shown that depletion of IL-22 in the recovery phase of the AOM/DSS model of colitis-associated cancer reduces tumor number and tumor score. ${ }^{25}$ However, to our knowledge it has not been determined whether IL-22 is essential for Hh-induced 
a

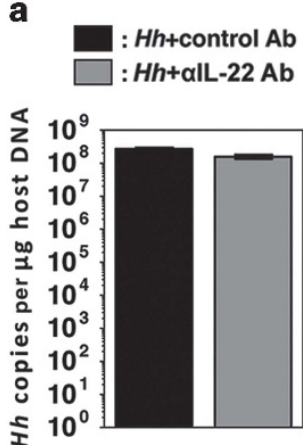

b

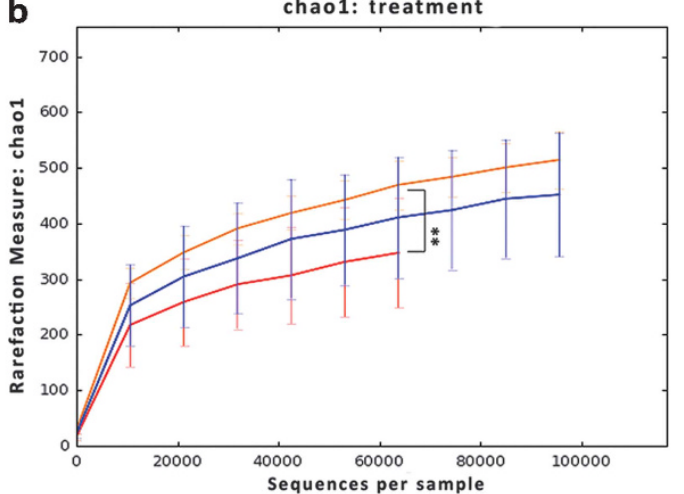

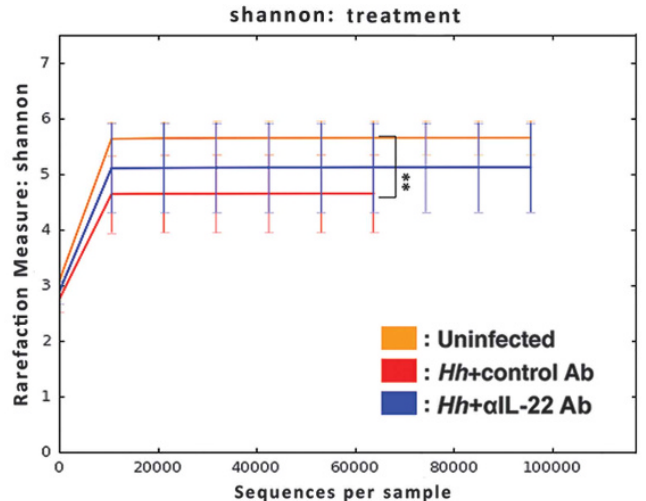

shannon: treatment

equences per sample
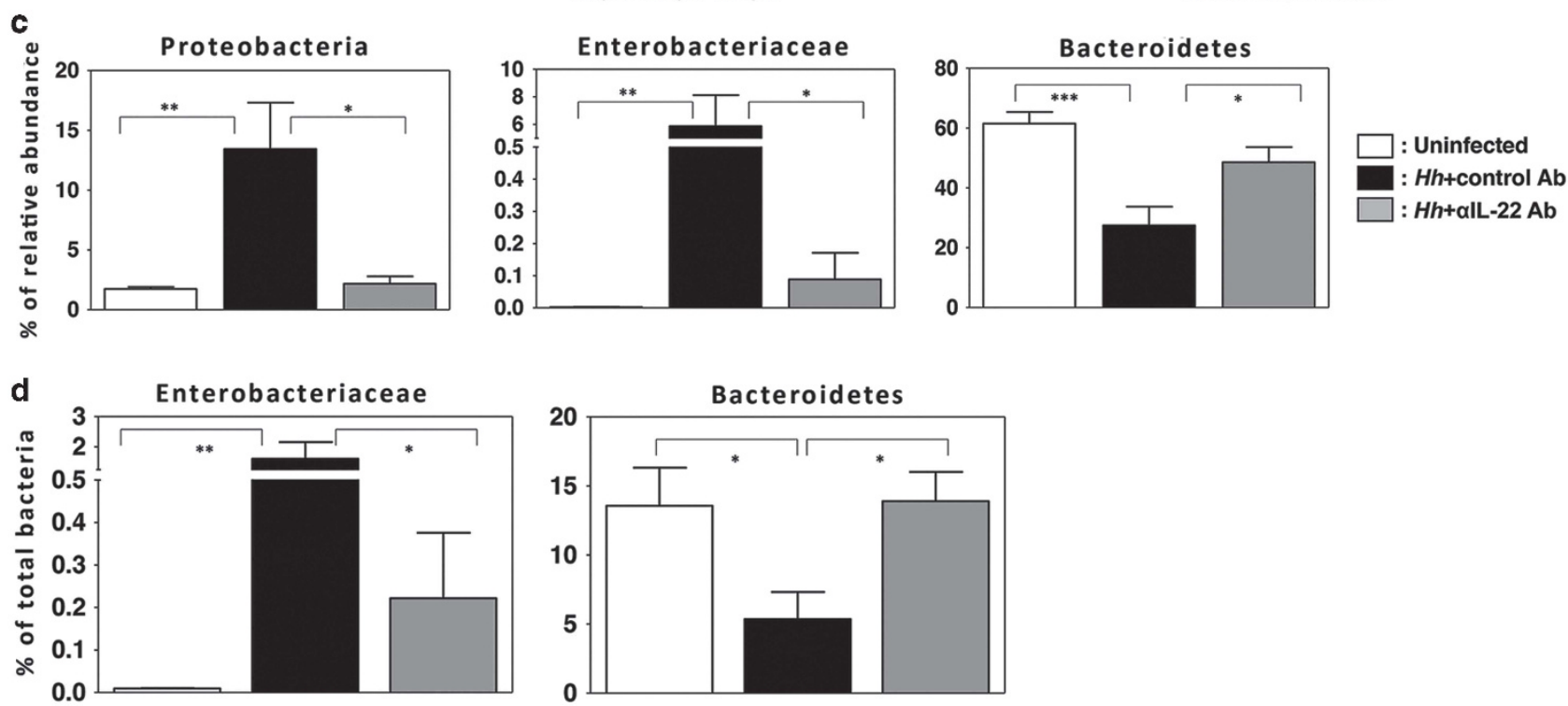

Figure $9 \mathrm{Hh}$ infection induces IL-22-dependent dysbiosis. (a) Quantification of Hh colonization density in the cecal content. $n=9-11$. (b) Chao and Shannon diversity indices from 16S rRNA sequencing data obtained from fecal pellets of uninfected mice and Hh-infected mice treated with control or antiIL-22 antibody as indicated. (c) Relative abundance of indicated taxa from groups described in $\mathbf{b}$. (d) Relative abundance of indicated taxa as determined by taxa-specific 16 s rRNA qPCR from groups described in $\mathbf{b} .{ }^{*} P<0.05,{ }^{\star *} P<0.01$, and ${ }^{\star * *} P<0.001$.

dysplasia that develops in the absence of carcinogen treatment. Our results strongly suggest that DNA damage is a central component of both the initial and chronic response to $\mathrm{Hh}$ infection even in the absence of an exogenous carcinogen, and that IL-22 plays a key role in this process. It has also been demonstrated that IL-22 plays an important role in the development of colon cancer in $\mathrm{APC}^{\mathrm{min} /+}$ mice that lacks overt signs of inflammation. ${ }^{25}$ However, whether induction of epithelial iNOS expression and the development of DSBs are associated with the development of cancer in this model will require further study.

Depletion of IL-22 also influenced the severity of Hh-induced inflammation. Two weeks after Hh infection IL-22 depletion did not significantly reduce the severity of inflammation or inflammatory cytokine production in the cecum, although it did reduce inflammation within the colon (Supplementary Figure 2). These results are consistent with the results of Morrison, who recently demonstrated that neutralization of IL-22 following infection of C57BL/6 mice with $\mathrm{Hh}$ and treatment with IL-10-receptor monoclonal antibody inhibited development of inflammation in the colon, but not the cecum. ${ }^{26}$ In contrast, 10 weeks after infection, depletion of IL-22 inhibited inflammation in both the cecum and colon consistent with the previous observations of Kirchberger et al. ${ }^{10}$

We have suggested that $\mathrm{Hh}$-induced NO production could be a central factor driving DNA damage and ultimately carcinogenesis in colitis-associated cancer. ${ }^{8}$ Here we demonstrate strong induction of iNOS activity at $2 \mathrm{WPI}$, which is largely confined to epithelial cells, rather than both epithelial cells and macrophages observed after chronic infection at 10 or 20 weeks. ${ }^{8}$ Further, we were quite surprised to observe crypt epithelial cells that stained positively for $\gamma \mathrm{H} 2 \mathrm{AX}$ within the cecum at $2 \mathrm{WPI}$, and to find that this staining depends on the function of iNOS. Given that $\gamma \mathrm{H} 2 \mathrm{AX}^{+}$epithelial cells were not observed within proliferating $\mathrm{Ki} \mathrm{F}^{+}$crypt epithelial cells from sham-infected mice (Figure 3a), it seems unlikely that induction of $\gamma \mathrm{H} 2 \mathrm{AX}^{+}$foci is a normal aspect of crypt epithelial cell proliferation, but rather specifically associated with $\mathrm{Hh}$ infection. Our results suggest that induction of DSBs results from $\mathrm{Hh}$-induced iNOS expression. 
One interesting aspects of our results is that while we observed virtually complete inhibition of DSBs by IL-22 depletion at 2 WPI, we noted only partial suppression at 10 WPI. This does not seem to reflect inability to suppress epithelial iNOS expression at the later time point as immunofluoresence demonstrated nearly complete suppression at both time points. It is possible that IL-22-independent expression of iNOS by infiltrating phagocytes at the later time point explains this partial response, and this would be consistent with our observation that depletion of IL-22 does not significantly inhibit iNOS mRNA expression within total RNA isolated from cecum or colon at $10 \mathrm{WPI}$, despite a marked inhibition of iNOS staining on intestinal epithelial cells. Certainly, additional experiments employing tissue-specific knockouts of the IL-22 receptor and iNOS will be necessary to help clarify these issues. An alternative explanation for this observation is that IL-22mediated inflammation damages intestinal stem cells and that these damaged stem cells produce progeny that are more susceptible to DNA DSBs. If correct, the observation of DSBs within crypt epithelial cells might be a marker for genetically unstable intestinal stem cells, a potential precursor for cancer. Further, if the intestinal stem cell is the key target of injury, one might anticipate that susceptibility to DNA damage would exhibit a clonal pattern. In fact, we believe that our results are consistent with a clonal process, in that at both 2 and 10 weeks epithelial DSBs were clustered in individual crypts rather than exhibiting a diffuse distribution. Our estimate that $5 \%$ of crypts exhibit DSBs at 2 WPI and $40 \%$ of crypts exhibit DSBs at 10 WPI is consistent with a clonal rather than diffuse pattern of injury. Further studies employing methodology that can trace descendants of individual ISCs will be necessary to unravel this issue.

A central tenet of the model proposed here is that NOmediated DSBs play an integral role in the development of colitis-associated cancer. However, studies directed at evaluating the role of iNOS in murine models of intestinal cancer are somewhat inconsistent. We have previously shown that treatment of Hh-infected 129RAG2 $2^{-1-}$ mice with NMA results in a trend toward reduced dysplasia scores in a chronic infection model. ${ }^{8}$ Ahn et al..$^{27}$ demonstrated a significant reduction in adenoma development within both the large and small bowel of $\mathrm{APC}^{\mathrm{min} /+}$ mice lacking iNOS. Shaked et al. ${ }^{28}$ demonstrated that both colonic tumors and epithelial DNA damage observed in $\operatorname{IKK} \beta(\mathrm{EE})^{\mathrm{IEC}} / \mathrm{APC}^{+/ \Delta \mathrm{IEC}}$ mice were reduced by treatment with NMA. These studies strongly support the concept that iNOS activity and NO-mediated epithelial DNA damage are key factors in the development of cancer of the lower bowel. In contrast, Zhang et al. ${ }^{29}$ have shown increased severity of dysplasia and increased number of polyps within the ascending colon of IL10 ${ }^{-1-} / \mathrm{iNOS}^{-1-}$ mice compared to IL $10^{-1-}$ mice after 6 months of age. While there may be many variables that explain the discrepancy between these model systems, it has been suggested that reduced nitrosative stress expected in iNOS-deficient mice is largely compensated for by induction of eNOS. ${ }^{30}$ Thus, further experiments are needed to fully understand the role of
iNOS and NO production in inflammatory-associated carcinogenesis.

This study confirms a central role for IL-22 in the regulation of anti-microbial peptide expression in the lower bowel following infection with $\mathrm{Hh}$. Further, we demonstrate here that infection with $\mathrm{Hh}$ rapidly induced a dysbiotic state characterized by expansion of Enterobacteriaceae that was partially reversed by depletion of IL-22. Dysbiosis, and especially expansion of Enterobacteriaceae, has been associated with both IBD and colon cancer. ${ }^{31-33}$ Previous studies have suggested that Enterobacteriaceae and especially certain selected E. coli strains express colibactin genotoxins. ${ }^{22}$ These toxins, like cytolethal distending toxin produced by $\mathrm{Hh}$, induce DNA damage and DSBs. ${ }^{23,34,35}$ Interestingly, using two different models of lower bowel inflammation, it has been demonstrated that iNOS-dependent nitrate production enables the relative expansion of $E$. coli strains, which can utilize nitrate as a terminal electron receptor for anaerobic respiration. ${ }^{36}$ Thus, it might be expected that IL-22-driven iNOS induction could lead to expansion of Enterobacteriaceae including those that carry genotoxins associated with cancer. In fact, we have observed the widespread presence of colibactin producing E. coli within our colony, ${ }^{37}$ and although not specifically quantified in this study, it is possible that Hh could promote DNA damage by enhancing colonization with these E. coli. The relationship between $\mathrm{Hh}$ infection, dysbiosis, genotoxins, and DNA damage will clearly require further studies. However, it is notable that a previous study observed increased numbers of $\gamma \mathrm{H} 2 \mathrm{AX}$-positive epithelial cells in AOM-treated $\mathrm{IL}-10^{-1-}$ mice mono-associated with colibactin producing E. coli, compared to mice mono-associated with an isogenic mutant that lacks the ability to produce colibactin. ${ }^{22}$ Thus, understanding the role of IL-22 in this process could lead to novel insights into the interface between dysbiosis, immune response, and cancer.

\section{METHODS}

Hh culture, animals, and in vivo infection. Hh strain $3 \mathrm{~B} 1$ (American Type Culture Collection 51449) was cultured as we have previously described. Six- to eight-week-old male and female 129S6/SvEvTacRag2 $2^{\text {tm1Fwa }}$ (129Rag $2^{-1-}$ ) mice obtained from in-house colonies free of Helicobacter species were orally dosed three times every other day with either $2 \times 10^{8} \mathrm{CFU}$ of $\mathrm{Hh}$ or sham dosed with Brucella broth. Mice were killed with $\mathrm{CO}_{2}$ at 2, 6, or 10 WPI. For IL-22 depletion experiments, infected mice received $100 \mu \mathrm{g}$ control Ab (GP120 10E7.1D2) or anti-IL-22 Ab (8E11) (Genentech, South San Francisco, CA) every other day during the last week of infection. For NMA treatment experiments, mice were treated with either $30 \mathrm{mM}$ L-NMMA (Enzo) or $30 \mathrm{mM}$ acetate (control) pH 7.0 in the drinking water for the final 7 days prior to necropsy. Mice were maintained under specific pathogen-free conditions within a facility accredited by the Association for the Assessment and Accreditation of Laboratory Animal Care. All animal experiments were approved by the Massachusetts Institute of Technology Committee on Animal Care.

Histology scoring of cecal and colon lesions. Cecum and colon obtained from mice were fixed in $10 \%$ formalin immediately after necropsy. Paraffin-embedded sections were prepared and $4 \mu \mathrm{m}$ sections were stained with hematoxylin and eosin. All slides were examined by board-certified veterinary pathologists blinded to sample 
identity. HAI is the sum of scoring of inflammation, edema, epithelial defects, crypt atrophy, hyperplasia, and dysplasia/neoplasia on a scale of 0-4 (maximum score 24 ). ${ }^{38}$

qPCR analysis of cytokine mRNA expression. Tissues for RNA isolation were collected immediately upon killing, snap-frozen in liquid nitrogen, and stored at $-80{ }^{\circ} \mathrm{C}$ prior to processing. RNA was prepared using Trizol reagents according to the manufacturer's instructions (Invitrogen, Grand Island, NY). Five microgram of total RNA from each sample was reverse transcribed using the HighCapacity cDNA Archive kit (Applied Biosystems, Foster City, CA). mRNA levels for Reg3 $\beta$, Reg3 $\gamma$, TNF, iNOS, and IL-22 were measured by $\mathrm{qPCR}$ using commercial primers and probes (TaqMan Gene Expression Assays) in the 7500 FAST Sequence Detection System. Transcript levels were compared using the $\Delta \Delta \mathrm{CT}$ method (Applied Biosystems).

Immunofluorescence staining. Five micrometer sections of formalin fixed paraffin-embedded tissue were deparaffinized and washed in gradient ethanol $100 \%, 90 \%, 70 \%$, and water. After antigen retrieval (boiling at $95^{\circ} \mathrm{C}$ for $20 \mathrm{~min}$ ) in Dako modified citrate-based buffer (Dako, S1700), sections were blocked with 3\% BSA in PBS-Triton $(0.03 \% \mathrm{v} / \mathrm{v})$ overnight, and then incubated with anti-EpCAM antibody (Santa Cruz Biotechnology, Dallas, TX, Cat\# sc53532), anti-iNOS antibody (Abcam, Cambridge, MA, Cat\#: ab15323), F4/80 antibody (Abcam, Cat\# ab6640), anti-nitrotyrosine antibody (Millipore, Billerica, MA, Cat\#: 05-233), anti-Ki67 antibody (BD Pharmingen, San Jose, CA, Cat\#: 550809), anti- $\gamma \mathrm{H} 2 \mathrm{AX}$ antibody (Cell Signaling, Danvers, MA, Cat\#: 9718), anti-Phospho-STAT3 (Tyr705) (Cell Signaling, Cat\#: 9145), and anti-MPO antibody (Abcam cat\#: ab45977) at room temperature for $1 \mathrm{~h}$. Primary antibodies were visualized with Alexa-Fluor-568 conjugated or Alexa-Fluor-488 conjugated secondary antibodies (goat-anti-rabbit, goat-anti-rat, or goat-anti-mouse, Invitrogen). Tissue sections were counter-stained with DAPI. All the stained slides were examined under a Zeiss Axioskop 2 plus microscope with QIClick digital CCD Camera (QImaging, Surrey, BC, Canada). Morphometric analysis from at least eight random images was performed using Image Pro-Plus (version 7.2, Media Cybematics, Silver Spring, MD). All images were acquired using a $\times 20$ or $\times 100$ objective lens.

Western blotting. Cecum samples were homogenized with a handheld homogenizer in $300 \mu$ l of RIPA buffer (Thermo Fisher, Waltham, MA) supplemented with complete protease inhibitors and phosSTOP phosphatase inhibitors (Roche, South San Francisco, CA). Five microgram of protein was subject to PAGE and blotted with antiGAPDH (Cell Signaling 2118), anti-STAT3 (Cell Signaling 4904), or anti-pSTAT3 (Y705) (Cell Signaling 9145).

Culture of mouse enteroids. Organoids were isolated and cultured by established methods. ${ }^{39}$ Organoid cultures were maintained and split weekly by repeated passage through a 25 -gauge needle. Newly passaged organoids were treated with PBS (untreated) or mIL-22 (GenScript) as noted. Organoids were collected after 3 days of treatment and homogenized in Trizol (Thermo Fisher) by passage through a 25-gauge needle. Organoid RNA was isolated by chloroform extraction followed by column purification (RNEasy Mini Kit, Qiagen) and $1 \mu \mathrm{g}$ reverse transcribed using the SuperScript Vilo cDNA synthesis Kit (Thermo Fisher). Two microliter of diluted cDNA was analyzed on an ABI StepOnePlus machine using Taqman qPCR probes as above.

Quantitative PCR for $\mathrm{Hh}$ colonization levels. To quantify $\mathrm{Hh}$ colonization levels, DNA was prepared from the cecal content using a High Pure PCR Template kit according to the manufacturer's protocol (Roche Applied Science). Levels of the Hh 16s rRNA gene were measured by qPCR in the 7500 Fast Sequence Detection System (Applied Biosystems) as described previously. ${ }^{23}$ The colonization levels of $\mathrm{Hh}$ were quantified by normalizing to $\mu \mathrm{g}$ of mouse chromosomal DNA measured by qPCR using $18 \mathrm{~S}$ rRNA gene-based primers and probe mixture (Life Technology, Waltham, MA).

Bacterial 16S rRNA sequencing and qPCR. DNA was extracted from fecal pellets using the UltraClean Fecal DNA Kit (MO BIO Laboratories, Carlsbad, CA). Amplicons were generated using oligonucleotide primers that target approximately $300 \mathrm{bp}$ of the V4 variable region of the $16 \mathrm{~S}$ rRNA gene (primers $515 \mathrm{~F}$ and $806 \mathrm{R})^{40}$ and also were barcoded and pooled to construct the sequencing library, followed by sequencing with an Illumina MiSeq instrument to generate paired-end $150 \times 150$ reads. The software package QIIME 1.7.0 was used to analyze, display, and generate figures of microbiome data using a previously defined method. ${ }^{41}$

Bacterial DNA of Enterobacteriaceae and Bacteriodetes from fecal content was quantified using six-point standard curves constructed with reference bacteria specific for each bacterial group measured by qPCR in the 7500 Fast Sequence Detection System (Applied Biosystems). All the reactions were set up in Fast SYBR Green Master Mix (Applied Biosystems) at $20 \mu \mathrm{l}$ total volume. Copy number of Enterobacteriaceae and Bacteriodetes was normalized to copy number of universal bacteria. The initialization step was $95^{\circ} \mathrm{C}$ for $10 \mathrm{~min}$, the amplification step had 40 cycles of $95^{\circ} \mathrm{C}$ for $10 \mathrm{~s}$ followed by optimal annealing temperature for $45 \mathrm{~s}$. The primers and reaction conditions are listed in Supplementary Table S1.

Statistical analyses. Statistical analyses were performed using the GraphPad Prism 5 software package (La Jolla, CA). Histopathologic scores were compared using a Mann-Whitney nonparametric $U$ test with median values shown. Cytokine expression and Hh colonization levels were analyzed using unpaired two-tailed Student's $t$-test, with mean value and s.e.m. shown. $P<0.05$ was considered statistically significant. ${ }^{*}{ }^{* *},{ }^{* * *}, * * * *$ indicate $P<0.05, P<0.01, P<0.001$, and $P<0.0001$, respectively.

SUPPLEMENTARY MATERIAL is linked to the online version of the paper at http://www.nature.com/mi

\section{ACKNOWLEDGMENTS}

We thank Wenjun Ouyang for providing anti-IL-22 antibody and its control antibody, Charles G. Knutson for the preparation of NMA, Zeli Shen for her assistance in preparation of $\mathrm{Hh}$, and Zhongming Ge and Yan Feng (Fox lab, MIT) for helpful discussion. Grant support: NIH T32-OD010978-26, R01OD011141, R01-DK099204, P01-CA028842-29, and P30-ES002109.

\section{AUTHOR CONTRIBUTIONS}

C.W. and G.G. study concept and design, acquisition of data, analysis and interpretation of data, and drafting of manuscript. E.B., D.A.P., H.H., A.S., and E.A.C. study concept and design, acquisition of data, and analysis and interpretation of data. S.M., N.M.A.P. and V.B. acquisition of data and analysis and interpretation of data. S.P.S. and C.S.W. study concept and design, acquisition of data, and analysis and interpretation of data. G.N.W. critical revision of manuscript for important intellectual content. S.R.T. and J.G.F. study concept and design, analysis and interpretation of data, critical revision of manuscript for important intellectual content, obtaining funding, and study supervision. B.H.H. study concept and design, analysis and interpretation of data, drafting of manuscript, obtaining funding, and study supervision.

\section{DISCLOSURE}

The authors declared no conflict of interest.

c) 2017 Society for Mucosal Immunology

\section{REFERENCES}

1. Kappelman, M.D. et al. The prevalence and geographic distribution of Crohn's disease and ulcerative colitis in the United States. Clin. Gastroenterol. Hepatol. 5, 1424-1429 (2007). 
2. Eaden, J.A., Abrams, K.R. \& Mayberry, J.F. The risk of colorectal cancer in ulcerative colitis: a meta-analysis. Gut 48, 526-535 (2001).

3. Canavan, C., Abrams, K.R. \& Mayberry, J. Meta-analysis: colorectal and small bowel cancer risk in patients with Crohn's disease. Aliment. Pharmacol. Ther. 23, 1097-1104 (2006).

4. Kiraly, O., Gong, G., Olipitz, W., Muthupalani, S. \& Engelward, B.P. Inflammation-induced cell proliferation potentiates DNA damage-induced mutations in vivo. PLoS Genet. 11, e1004901 (2015).

5. Davis, C.D. \& Milner, J.A. Gastrointestinal microflora, food components and colon cancer prevention. J. Nutr. Biochem. 20, 743-752 (2009).

6. Sobhani, I. et al. Microbial dysbiosis and colon carcinogenesis: could colon cancer be considered a bacteria-related disease?. Therap. Adv. Gastroenterol. 6, 215-229 (2013).

7. Fox, J.G., Ge, Z., Whary, M.T., Erdman, S.E. \& Horwitz, B.H. Helicobacter hepaticus infection in mice: models for understanding lower bowel inflammation and cancer. Mucosal Immunol. 4, 22-30 (2011).

8. Erdman, S.E. et al. Nitric oxide and TNF- $\alpha$ trigger colonic inflammation and carcinogenesis in Helicobacter hepaticus-infected, Rag2-deficient mice. Proc. Natl Acad. Sci. USA 106, 1027-1032 (2009).

9. Erdman, S.E. et al. $\mathrm{CD} 4{ }^{+} \mathrm{CD} 25^{+}$regulatory $\mathrm{T}$ lymphocytes inhibit microbially induced colon cancer in Rag2-deficient mice. Am. J. Pathol. 162, 691-702 (2003).

10. Kirchberger, S. et al. Innate lymphoid cells sustain colon cancer through production of interleukin-22 in a mouse model. J. Exp. Med. 210, 917-931 (2013).

11. Knutson, C.G. et al. Chemical and cytokine features of innate immunity characterize serum and tissue profiles in inflammatory bowel disease. Proc. Natl Acad. Sci. USA 110, E2332-E2341 (2013).

12. Nagalakshmi, M.L., Rascle, A., Zurawski, S., Menon, S. \& de Waal Malefyt, R. Interleukin-22 activates STAT3 and induces IL-10 by colon epithelial cells. Int. Immunopharmacol. 4, 679-691 (2004).

13. Zheng, Y. et al. Interleukin-22 mediates early host defense against attaching and effacing bacterial pathogens. Nat. Med. 14, 282-289 (2008).

14. Zenewicz, L.A. et al. IL-22 deficiency alters colonic microbiota to be transmissible and colitogenic. J. Immunol. 190, 5306-5312 (2013).

15. Mangerich, A. et al. Infection-induced colitis in mice causes dynamic and tissue-specific changes in stress response and DNA damage leading to colon cancer. Proc. Natl Acad. Sci. USA 109, E1820-E1829 (2012).

16. Gal, A., Tamir, S., Kennedy, L.J., Tannenbaum, S.R. \& Wogan, G.N. Nitrotyrosine formation, apoptosis, and oxidative damage: relationships to nitric oxide production in SJL mice bearing the RcsX tumor. Cancer Res. 57, 1823-1828 (1997).

17. Seril, D.N., Liao, J., Yang, G.Y. \& Yang, C.S. Oxidative stress and ulcerative colitis-associated carcinogenesis: studies in humans and animal models. Carcinogenesis 24, 353-362 (2003).

18. Rogakou, E.P., Pilch, D.R., Orr, A.H., Ivanova, V.S. \& Bonner, W.M. DNA double-stranded breaks induce histone H2AX phosphorylation on serine 139. J. Biol. Chem. 273, 5858-5868 (1998).

19. Xie, Q.W., Kashiwabara, Y. \& Nathan, C. Role of transcription factor NF-kappa B/Rel in induction of nitric oxide synthase. J. Biol. Chem. 269, 4705-4708 (1994).

20. Ziesche, E., Bachmann, M., Kleinert, H., Pfeilschifter, J. \& Muhl, H. The interleukin-22/STAT3 pathway potentiates expression of inducible nitricoxide synthase in human colon carcinoma cells. J. Biol. Chem. 282, 16006-16015 (2007).

21. Boulard, O., Kirchberger, S., Royston, D.J., Maloy, K.J. \& Powrie, F.M. Identification of a genetic locus controlling bacteria-driven colitis and associated cancer through effects on innate inflammation. J. Exp. Med. 209, 1309-1324 (2012).

22. Arthur, J.C. et al. Intestinal inflammation targets cancer-inducing activity of the microbiota. Science 338, 120-123 (2012).

23. Ge, Z. et al. Cytolethal distending toxin is essential for Helicobacter hepaticus colonization in outbred Swiss Webster mice. Infect. Immun. 73, 3559-3567 (2005).

24. Jobin, C. Colorectal cancer: looking for answers in the microbiota. Cancer Discov. 3, 384-387 (2013).

25. Huber, $\mathrm{S}$. et al. IL-22BP is regulated by the inflammasome and modulates tumorigenesis in the intestine. Nature 491, 259-263 (2012).

26. Morrison, P.J. et al. Differential requirements for IL-17A and IL-22 in cecal versus colonic inflammation induced by Helicobacter hepaticus. Am. J. Pathol. 185, 3290-3303 (2015).

27. Ahn, B. \& Ohshima, H. Suppression of intestinal polyposis in $\mathrm{Apc}_{(\mathrm{Min}} /+$ ) mice by inhibiting nitric oxide production. Cancer Res. 61, 8357-8360 (2001).

28. Shaked, H. et al. Chronic epithelial NF-kB activation accelerates APC loss and intestinal tumor initiation through iNOS up-regulation. Proc. Natl Acad. Sci. USA 109, 14007-14012 (2012).

29. Zhang, R., Ma, A., Urbanski, S.J. \& McCafferty, D.M. Induction of inducible nitric oxide synthase: a protective mechanism in colitis-induced adenocarcinoma. Carcinogenesis 28, 1122-1130 (2007).

30. Seril, D.N., Liao, J. \& Yang, G.Y. Colorectal carcinoma development in inducible nitric oxide synthase-deficient mice with dextran sulfate sodiuminduced ulcerative colitis. Mol. Carcinog. 46, 341-353 (2007).

31. Allali, I. et al. Gut microbiome compositional and functional differences between tumor and non-tumor adjacent tissues from cohorts from the US and Spain. Gut Microbes 6, 161-172 (2015).

32. Couturier-Maillard, A. et al. NOD2-mediated dysbiosis predisposes mice to transmissible colitis and colorectal cancer. J. Clin. Invest. 123, 700-711 (2013).

33. Haberman, Y. et al. Pediatric Crohn disease patients exhibit specific ileal transcriptome and microbiome signature. J. Clin. Invest. 124, 3617-3633 (2014).

34. Buc, E. et al. High prevalence of mucosa-associated E. coli producing cyclomodulin and genotoxin in colon cancer. PLoS One 8, e56964 (2013).

35. Cuevas-Ramos, G., Petit, C.R., Marcq, I., Boury, M., Oswald, E. \& Nougayrede, J.P. Escherichia coli induces DNA damage in vivo and triggers genomic instability in mammalian cells. Proc. Natl Acad. Sci. USA 107, 11537-11542 (2010).

36. Winter, S.E. et al. Host-derived nitrate boosts growth of E. coli in the inflamed gut. Science 339, 708-711 (2013).

37. Garcia, A. et al. Cytotoxic Escherichia coli strains encoding colibactin colonize laboratory mice. Microbes Infect. 18, 777-786 (2016).

38. Rogers, A.B. \& Houghton, J. Helicobacter-based mouse models of digestive system carcinogenesis. Methods Mol. Biol. 511, 267-295 (2009).

39. Mahe, M.M. et al. Establishment of Gastrointestinal Epithelial Organoids. Curr. Protoc. Mouse Biol. 3, 217-240 (2014).

40. Caporaso, J.G. et al. Global patterns of $16 \mathrm{~S}$ rRNA diversity at a depth of millions of sequences per sample. Proc. Natl Acad. Sci. USA 108 (Suppl 1), 4516-4522 (2010).

41. Swennes, A.G. et al. Helicobacter hepaticus infection promotes hepatitis and preneoplastic foci in farnesoid X receptor (FXR) deficient mice. PLoS One 9, e106764 (2014). 